\title{
Hyers-Ulam stability of a certain Fredholm integral equation $\rrbracket^{*}$
}

\author{
Alberto SIMÕES ${ }^{1,2}$, Ponmana SELVAN ${ }^{3}$ \\ ${ }^{1}$ CMA-UBI, Center of Mathematics and Applications \\ University of Beira-Interior, Covilhã, 6201-001, Portugal \\ ${ }^{2}$ CIDMA, Center for Research and Development in Mathematics and Applications \\ University of Aveiro, Aveiro, 3810-193, Portugal \\ ${ }^{3}$ Department of Mathematics, Sri Sai Ram Institute of Technology, \\ Tamil Nadu, Chennai, India
}

\begin{abstract}
In this paper, by using Fixed point Theorem we establish the Hyers-Ulam stability and Hyers-Ulam-Rassias stability of certain homogeneous Fredholm Integral equation of the second kind

$$
\phi(x)=\lambda \int_{0}^{1}(1+x+t) \phi(t) d t
$$

and the non-homogeneous equation

$$
\phi(x)=x+\lambda \int_{0}^{1}(1+x+t) \phi(t) d t
$$

for all $x \in[0,1]$ and $0<\lambda<\frac{2}{5}$.

AMS Mathematics Subject Classification: 26D10, 31K20, 39A10, 34A40, 34K20. Key Words: Hyers-Ulam Stability, Hyers-Ulam-Rassias stability, Fredholm Integral equation of second kind, Fixed point Theorem.
\end{abstract}

\section{Introduction}

The Ulam stability problem for various functional equation was initiated by S.M. Ulam 31] in 1940. Then, in the next year, D.H. Hyers [16] was solved the Ulam problem for Cauchy additive functional equation on Banach spaces. After that Aoki [3], Bourgin [6] and Rassias [25] have generalized the Hyers result. These days the Hyers-Ulam stability for different functional equations was proved by many mathematicians (see [4, 11, 26, 5]). A generalization Ulam problem was recently proposed by replacing functional equations with differential equations. In 1998, Alsina et al., [1] was proved the Hyers-Ulam stability of differential equation of first order of the form $y^{\prime}(t)=y(t)$. This result was generalized

*Accepted author's manuscript (AAM) of [Alberto SIMÕES, Ponmana SELVAN: Turkish Journal of Mathematics, 46: 87-98, doi:10.3906/mat-2106-120] The final publication is available at https://journals.tubitak.gov.tr/math/issues/mat-22-46-1/mat-46-1-7-2106-120.pdf 
by Takahasi [30] for Banach space valued differential equation $y^{\prime}(t)=\lambda y(t)$. Then several researchers have studied the Hyers-Ulam stability of differential equations in various directions, for example (see [7, 10, 18, 17, 19, 21, 20, 23, 22, 24, 29, 32]).

Now a days, the Hyers-Ulam stability of integral equations has been given attention. In 2015, L. Hua et al., [15] studied the Hyers-Ulam stability of some kinds of Fredholm integral equations. Also, in 2015, Z. Gu and J. Huang [14] are investigated the Hyers-Ulam stability of the Fredholm integral equation

$$
\phi(x)=f(x)+\lambda \int_{a}^{b} K(x, s) \phi(s) d s
$$

by fixed point Theorem. Recently, only few authors are investigating the Hyers-Ulam stability of the various integral equations (see [2, 8, 9, 12, 13, 27, 28]). Motivated by the above ideas, our foremost aim is to study the Hyers-Ulam stability and Hyers-Ulam-Rassias stability of the certain Fredholm Integral equations of second kind

$$
\phi(x)=\lambda \int_{0}^{1}(1+x+t) \phi(t) d t
$$

and

$$
\phi(x)=x+\lambda \int_{0}^{1}(1+x+t) \phi(t) d t
$$

for all $x \in[0,1]$ and $0<\lambda<\frac{2}{5}$ in the sense of Z. Gu and J. Huang [14].

\section{Preliminaries}

The following Theorems and Definitions are very useful to prove our main results.

Theorem 1. (Fixed Point Theorem) Let $(X, \rho)$ be a complete metric space. Assume that $T: X \rightarrow X$ is a strictly contractive operator with $\rho(T x, T y) \leq \theta \rho(x, y)$ where $0<\theta<1$. Then

(i) there exists an unique fixed point $x^{*}$ of $T$;

(ii) the sequence $\left\{T^{n} x\right\}_{n \in \mathbb{N}}$ converges to the fixed point $x^{*}$ of $T$.

Theorem 2. (Hölder's Inequality) Let $p>1, \frac{1}{p}+\frac{1}{q}=1, x \in L^{p}(E)$ and $y \in L^{q}(E)$. Then $x y \in L(E)$ and

$$
\int_{E}|x(t) y(t)| d t \leq\left(\int_{E}\left|x^{p}(t)\right| d t\right)^{\frac{1}{p}}\left(\int_{E}\left|y^{q}(t)\right| d t\right)^{\frac{1}{q}} .
$$

Now, we give the definition of Hyers-Ulam stability and Hyers-Ulam-Rassias stability of the Fredholm integral equations (1) and (2).

Definition 3. We say that the Fredholm integral equations (1) has the Hyers-Ulam stability, if there exists a real constant $S$ which satisfies the following conditions: For every $\epsilon>0$, and for each solution $\phi:[0,1] \rightarrow \mathbb{R}$ satisfying the inequation

$$
\left|\phi(x)-\lambda \int_{0}^{1}(1+x+t) \phi(t) d t\right| \leq \epsilon,
$$

then there is some $\psi:[0,1] \rightarrow \mathbb{R}$ satisfying the integral equation (1) such that

$$
|\phi(x)-\psi(x)| \leq S \epsilon, \quad \forall x \in[0,1] .
$$


Definition 4. We say that the Fredholm integral equations (2) has the Hyers-Ulam stability, if there exists a real constant $S$ which satisfies the following conditions: For every $\epsilon>0$, and for each solution $\phi:[0,1] \rightarrow \mathbb{R}$ satisfying the inequality

$$
\left|\phi(x)-x-\lambda \int_{0}^{1}(1+x+t) \phi(t) d t\right| \leq \epsilon,
$$

then there exists a solution $\psi:[0,1] \rightarrow \mathbb{R}$ satisfies the integral equation $(2)$ such that

$$
|\phi(x)-\psi(x)| \leq S \epsilon, \quad \forall x \in[0,1] .
$$

Definition 5. The Fredholm integral equations (1) is said to have the Hyers-Ulam-Rassias stability, if there exists a real constant $S$ which fulfill the following: For every $\theta \in$ $C\left(\mathbb{R}_{+}, \mathbb{R}_{+}\right)$, and for each solution $\phi:[0,1] \rightarrow \mathbb{R}$ satisfying the inequality

$$
\left|\phi(x)-\lambda \int_{0}^{1}(1+x+t) \phi(t) d t\right| \leq \theta(x),
$$

then there is a solution $\psi:[0,1] \rightarrow \mathbb{R}$ satisfying the integral equation (1) such that

$$
|\phi(x)-\psi(x)| \leq S \theta(x), \quad \forall x \in[0,1] .
$$

Definition 6. We say that the Fredholm integral equations (2) has the Hyers-Ulam-Rassias stability, if there exists a real constant $S$ which fulfill the following properties: For every $\theta \in C\left(\mathbb{R}_{+}, \mathbb{R}_{+}\right)$, and for each solution $\phi:[0,1] \rightarrow \mathbb{R}$ satisfying the inequation

$$
\left|\phi(x)-x-\lambda \int_{0}^{1}(1+x+t) \phi(t) d t\right| \leq \theta(x),
$$

then there exists some $\psi:[0,1] \rightarrow \mathbb{R}$ satisfying the integral equation (2) such that

$$
|\phi(x)-\psi(x)| \leq S \theta(x), \quad \forall x \in[0,1] .
$$

\section{Main Results}

In this section, we are going to prove the Hyers-Ulam stability and the Hyers-Ulam-Rassias stability of the homogeneous and non-homogeneous Fredholm integral equations of second kind (1) and (2) with $\lambda<\frac{2}{5}$. First, we investigate the two stabilities of the homogeneous Fredholm integral equation of second kind (1).

Theorem 7. Consider $H$ a fixed real number such that $H \geq \frac{5}{2}$ and $\lambda H<1$. Let $\phi:[0,1] \rightarrow \mathbb{R}$ a continuous function and the kernel $K:[0,1] \times[0,1] \rightarrow \mathbb{R}$ defined by $K(x, t)=1+x+t$. If $\phi$ is such that

$$
\left|\phi(x)-\lambda \int_{0}^{1}(1+x+t) \phi(t) d t\right| \leq \epsilon
$$

where $\epsilon \geq 0$ then there exists a solution $\psi:[0,1] \rightarrow \mathbb{R}$ of the Fredholm integral equation (1) and a real constant $S$ such that $|\phi(x)-\psi(x)| \leq S \epsilon$ for all $x \in[0,1]$.

Proof. Firstly, we define an operator $T$ by,

$$
(T \phi)(x)=\lambda \int_{0}^{1}(1+x+t) \phi(t) d t, \quad \phi \in L^{2}([0,1]) .
$$


We have for each $x \in[0,1]$,

$$
\left|\int_{0}^{1}(1+x+t) d t\right| \leq H \quad \text { and } \quad\left|\left(\int_{0}^{1} \int_{0}^{1}(1+x+t)^{2} d t d x\right)^{\frac{1}{2}}\right| \leq H,
$$

for any $H \geq \frac{5}{2}$.

Now, we define a metric $\rho$ as follows,

$$
\rho\left(\varphi_{1}, \varphi_{2}\right)=\left\{\left(\int_{0}^{1}\left|\frac{\varphi_{1}(x)-\varphi_{2}(x)}{\lambda H}\right|^{2} d x\right)^{\frac{1}{2}}: \varphi_{1}, \varphi_{2} \in L^{2}([0,1]), \lambda H<1\right\} .
$$

By using the Hölder's inequality, we obtain that

$$
\begin{aligned}
\int_{0}^{1}\left|\int_{0}^{1}(1+x+t) \phi(t) d t\right|^{2} d x & \leq \int_{0}^{1}\left(\int_{0}^{1}(1+x+t)^{2} d t \int_{0}^{1} \phi^{2}(t) d t\right) d x \\
& \leq \int_{0}^{1} \phi^{2}(t) d t \int_{0}^{1} \int_{0}^{1}(1+x+t)^{2} d t d x<\infty
\end{aligned}
$$

This implies that $T \phi \in L^{2}([0,1])$ and $T$ is a self-mapping of $L^{2}([0,1])$. Thus, the solution of the equation (4) is the fixed point of $T$. So,

$$
\begin{aligned}
\rho\left(T \varphi_{1}, T \varphi_{2}\right) & =\left(\int_{0}^{1}\left|\frac{\left(T \varphi_{1}\right)(x)-\left(T \varphi_{2}\right)(x)}{\lambda H}\right|^{2} d x\right)^{\frac{1}{2}} \\
& =\frac{1}{H}\left(\int_{0}^{1}\left|\int_{0}^{1}(1+x+t)\left(\varphi_{1}(t)-\varphi_{2}(t)\right) d t\right|^{2} d x\right)^{\frac{1}{2}} \\
& \leq \frac{1}{H}\left(\int_{0}^{1} \int_{0}^{1}(1+x+t)^{2} d t d x\right)^{\frac{1}{2}}\left(\int_{0}^{1}\left|\varphi_{1}(t)-\varphi_{2}(t)\right|^{2} d t\right)^{\frac{1}{2}} \\
& \leq\left(\int_{0}^{1}\left|\varphi_{1}(t)-\varphi_{2}(t)\right|^{2} d t\right)^{\frac{1}{2}} \\
& =\lambda H\left(\int_{0}^{1}\left|\frac{\varphi_{1}(t)-\varphi_{2}(t)}{\lambda H}\right|^{2} d t\right)^{\frac{1}{2}} \\
& =\lambda H \rho\left(\varphi_{1}, \varphi_{2}\right) .
\end{aligned}
$$

Since $\lambda H<1, T$ is a strictly contractive operator. Then by Theorem 1 the equation (4) has a unique solution $\phi^{*} \in L^{2}([0,1])$, where $\phi^{*}=\lim _{r \rightarrow \infty} \phi_{r}$ for

$$
\phi_{r}(x)=\lambda \int_{0}^{1}(1+x+t) \phi_{r-1}(t) d t
$$

and $\phi_{0} \in L^{2}([0,1])$ is an arbitrary function.

Let $\psi \in L^{2}([0,1])$ be a solution of inequality (3) and

$$
\psi(x)-\lambda \int_{0}^{1}(1+x+t) \psi(t) d t=: h(x) .
$$

Obviously, we have $|h(x)| \leq \epsilon$ for all $x \in[0,1]$. Then we can conclude that the solution of equation

$$
\psi(x)=h(x)+\lambda \int_{0}^{1}(1+x+t) \psi(t) d t
$$


is $\psi^{*} \in L^{2}([0,1])$, where $\psi^{*}=\lim _{r \rightarrow \infty} \psi_{r}$ for

$$
\psi_{r}(x)=h(x)+\lambda \int_{0}^{1}(1+x+t) \psi_{r-1}(t) d t
$$

and $\psi_{0} \in L^{2}([0,1])$ is an arbitrary function.

For $\phi_{0}(x)=\psi_{0}(x)=0$, we get,

$$
\begin{aligned}
\left|\phi_{1}(x)-\psi_{1}(x)\right|= & |h(x)| \leq \epsilon \\
\left|\phi_{2}(x)-\psi_{2}(x)\right|= & \left|h(x)+\lambda \int_{0}^{1}(1+x+t)\left(\psi_{1}(t)-\phi_{1}(t)\right) d t\right| \leq \epsilon\left(1+\lambda \int_{0}^{1}|1+x+t| d t\right) \\
\left|\phi_{3}(x)-\psi_{3}(x)\right|= & \left|h(x)+\lambda \int_{0}^{1}\left(1+x+t_{2}\right)\left(\psi_{2}\left(t_{2}\right)-\phi_{2}\left(t_{2}\right)\right) d t_{2}\right| \\
\leq & \epsilon+\epsilon \lambda \int_{0}^{1}\left|1+x+t_{2}\right|\left(1+\lambda \int_{0}^{1}\left|1+t_{2}+t_{1}\right| d t_{1}\right) d t_{2} \\
\leq & \epsilon\left(1+\lambda \int_{0}^{1}\left|1+x+t_{2}\right| d t_{2}+\lambda^{2} \int_{0}^{1}\left|1+x+t_{2}\right| \int_{0}^{1}\left|1+t_{2}+t_{1}\right| d t_{1} d t_{2}\right) \\
\ldots & \ldots \ldots \int_{0}^{1}(1+x+t)\left(\psi_{r-1}(x)-\phi_{r-1}(x)\right) d t \mid \\
\left|\phi_{r}(x)-\psi_{r}(x)\right|= & |h(x)+\lambda| \\
\leq & \epsilon\left(1+\lambda \int_{0}^{1}\left|1+x+t_{r-1}\right| d t_{r-1}\right. \\
& +\lambda^{2} \int_{0}^{1}\left|1+x+t_{r-1}\right| \int_{0}^{1}\left|1+t_{r-1}+t_{r-2}\right| d t_{r-2} d t_{r-1}+\cdots \\
& \ldots+\lambda^{r-1} \int_{0}^{1}\left|1+x+t_{r-1}\right| \int_{0}^{1}\left|1+t_{r-1}+t_{r-2}\right| \int_{0}^{1}\left|1+t_{r-2}+t_{r-3}\right| \cdots \\
& \left.\ldots \int_{0}^{1}\left|1+t_{2}+t_{1}\right| d t_{1} \ldots d t_{r-3} d t_{r-2} d t_{r-1}\right) \\
\leq & \epsilon\left(1+\lambda H+(\lambda H)^{2}+\ldots+(\lambda H)^{r-1}\right)=\epsilon\left(\frac{1-(\lambda H)^{r}}{1-\lambda H}\right), \\
& (1+\ldots i n
\end{aligned}
$$

as $r \rightarrow \infty$, we obtain

$$
\left|\phi^{*}(x)-\psi^{*}(x)\right| \leq \frac{1}{1-\lambda H} \epsilon .
$$

Let us choose $S=\frac{1}{1-\lambda H}$, hence $\left|\phi^{*}(x)-\psi^{*}(x)\right| \leq S \epsilon$, and $0<\lambda H<1$, where $S$ is the Hyers-Ulam stability constant for (1). Hence, by the virtue of Definition 3 the Fredholm integral equation (1) has the Hyers-Ulam stability.

The following theorem shows the Hyers-Ulam-Rassias stability of the homogeneous Fredholm integral equation of second kind (1).

Theorem 8. Consider $H$ a fixed real number such that $H \geq \frac{5}{2}$ and $\lambda H<1$. Let $\phi:[0,1] \rightarrow \mathbb{R}$ a continuous function and the kernel $K:[0,1] \times[0,1] \rightarrow \mathbb{R}$ defined by $K(x, t)=1+x+t$ such that

$$
\int_{0}^{1}|1+x+t| \theta(t) d t \leq \theta(x) \int_{0}^{1}|1+x+t| d t
$$


for all $x \in[0,1]$, where $\theta \in C\left(\mathbb{R}_{+}, \mathbb{R}_{+}\right)$. If $\phi$ is such that

$$
\left|\phi(x)-\lambda \int_{0}^{1}(1+x+t) \phi(t) d t\right| \leq \theta(x)
$$

then there exists a solution $\psi:[0,1] \rightarrow \mathbb{R}$ of the Fredholm integral equation (1) and a real constant $S$ such that $|\phi(x)-\psi(x)| \leq S \theta(x)$ for all $x \in[0,1]$.

Proof. By a similar procedure to the previous we define a strictly contractive operator $T$ as in (4) since $\lambda H<1$. By (5) we have $|h(x)| \leq \theta(x)$ for all $x \in[0,1]$. As in the previous proof, for $\phi_{0}(x)=\psi_{0}(x)=0$, we get,

$$
\begin{aligned}
\left|\phi_{1}(x)-\psi_{1}(x)\right|= & |h(x)| \leq \theta(x), \\
\left|\phi_{2}(x)-\psi_{2}(x)\right|= & \left|h(x)+\lambda \int_{0}^{1}(1+x+t)\left(\psi_{1}(t)-\phi_{1}(t)\right) d t\right| \leq \theta(x)\left(1+\lambda \int_{0}^{1}|1+x+t| d t\right) \\
\left|\phi_{3}(x)-\psi_{3}(x)\right|= & \left|h(x)+\lambda \int_{0}^{1}\left(1+x+t_{2}\right)\left(\psi_{2}\left(t_{2}\right)-\phi_{2}\left(t_{2}\right)\right) d t_{2}\right| \\
\leq & \theta(x)+\theta(x) \lambda \int_{0}^{1}\left|1+x+t_{2}\right|\left(1+\lambda \int_{0}^{1}\left|1+t_{2}+t_{1}\right| d t_{1}\right) d t_{2} \\
\leq & \theta(x)\left(1+\lambda \int_{0}^{1}\left|1+x+t_{2}\right| d t_{2}+\lambda^{2} \int_{0}^{1}\left|1+x+t_{2}\right| \int_{0}^{1}\left|1+t_{2}+t_{1}\right| d t_{1} d t_{2}\right) \\
\ldots & \ldots \ldots \\
\left|\phi_{r}(x)-\psi_{r}(x)\right|= & \left|h(x)+\lambda \int_{0}^{1}(1+x+t)\left(\psi_{r-1}(x)-\phi_{r-1}(x)\right) d t\right| \\
\leq & \theta(x)\left(1+\lambda \int_{0}^{1}\left|1+x+t_{r-1}\right| d t_{r-1}\right. \\
& +\lambda^{2} \int_{0}^{1}\left|1+x+t_{r-1}\right| \int_{0}^{1}\left|1+t_{r-1}+t_{r-2}\right| d t_{r-2} d t_{r-1}+\cdots \\
& \ldots+\lambda^{r-1} \int_{0}^{1}\left|1+x+t_{r-1}\right| \int_{0}^{1}\left|1+t_{r-1}+t_{r-2}\right| \int_{0}^{1}\left|1+t_{r-2}+t_{r-3}\right| \cdots \\
& \left.\ldots \int_{0}^{1}\left|1+t_{2}+t_{1}\right| d t_{1} \ldots d t_{r-3} d t_{r-2} d t_{r-1}\right) \\
\leq & \theta(x)\left(1+\lambda H+(\lambda H)^{2}+\ldots+(\lambda H)^{r-1}\right)=\theta(x)\left(\frac{1-(\lambda H)^{r}}{1-\lambda H}\right),
\end{aligned}
$$

as $r \rightarrow \infty$, we obtain

$$
\left|\phi^{*}(x)-\psi^{*}(x)\right| \leq \frac{1}{1-\lambda H} \theta(x)
$$

for all $x \in[0,1]$. Let us choose $S=\frac{1}{1-\lambda H}$, hence $\left|\phi^{*}(x)-\psi^{*}(x)\right| \leq S \theta(x)$, and $0<\lambda H<1$. Hence, by the virtue of Definition 5 the Fredholm integral equation (1) has the Hyers-Ulam-Rassias stability.

Now, we are going to establish the Hyers-Ulam stability of the non-homogeneous Fredholm integral equation of second kind (2). 
Theorem 9. Consider $H$ a fixed real number such that $H \geq \frac{5}{2}$ and $\lambda H<1$. Let $\phi:[0,1] \rightarrow \mathbb{R}$ a continuous function and the kernel $K:[0,1] \times[0,1] \rightarrow \mathbb{R}$ defined by $K(x, t)=1+x+t$. If $\phi$ is such that

$$
\left|\phi(x)-x-\lambda \int_{0}^{1}(1+x+t) \phi(t) d t\right| \leq \epsilon,
$$

where $\epsilon \geq 0$ then there exists a solution $\psi:[0,1] \rightarrow \mathbb{R}$ of the non-homogeneous Fredholm integral equation (2) and a real constant $S$ such that $|\phi(x)-\psi(x)| \leq S \epsilon$ for all $x \in[0,1]$.

Proof. Let us define an operator $T$ as

$$
(T \phi)(x)=x+\lambda \int_{0}^{1}(1+x+t) \phi(t) d t, \quad \phi \in L^{2}([0,1]) .
$$

We have $T \phi \in L^{2}([0,1])$ and $T$ a self-mapping of $L^{2}([0,1])$. The solution of the equation (8) is the fixed point of the strictly contractive operator $T$ since $\lambda H<1$. By Theorem 1 the equation (8) has a unique solution $\phi^{*} \in L^{2}([0,1])$, where $\phi^{*}=\lim _{r \rightarrow \infty} \phi_{r}$ for

$$
\phi_{r}(x)=x+\lambda \int_{0}^{1}(1+x+t) \phi_{r-1}(t) d t
$$

and $\phi_{0} \in L^{2}([0,1])$ is an arbitrary function.

Let $\psi \in L^{2}([0,1])$ be a solution of inequality (4) and

$$
\psi(x)-x-\lambda \int_{0}^{1}(1+x+t) \psi(t) d t=: h(x) .
$$

We have $|h(x)| \leq \epsilon$ for all $x \in[0,1]$. Then we can conclude that the solution of equation

$$
\psi(x)=h(x)+x+\lambda \int_{0}^{1}(1+x+t) \psi(t) d t
$$

is $\psi^{*} \in L^{2}([0,1])$, where $\psi^{*}=\lim _{r \rightarrow \infty} \psi_{r}$ for

$$
\psi_{r}(x)=h(x)+x+\lambda \int_{0}^{1}(1+x+t) \psi_{r-1}(t) d t
$$

and $\psi_{0} \in L^{2}([0,1])$ is an arbitrary function.

For $\phi_{0}(x)=\psi_{0}(x)=0$, we get,

$$
\begin{aligned}
\left|\phi_{r}(x)-\psi_{r}(x)\right|= & \left|h(x)+\lambda \int_{0}^{1}(1+x+t)\left(\psi_{r-1}(x)-\phi_{r-1}(x)\right) d t\right| \\
\leq & \epsilon\left(1+\lambda \int_{0}^{1}\left|1+x+t_{r-1}\right| d t_{r-1}\right. \\
& +\lambda^{2} \int_{0}^{1}\left|1+x+t_{r-1}\right| \int_{0}^{1}\left|1+t_{r-1}+t_{r-2}\right| d t_{r-2} d t_{r-1}+\cdots \\
& \cdots+\lambda^{r-1} \int_{0}^{1}\left|1+x+t_{r-1}\right| \int_{0}^{1}\left|1+t_{r-1}+t_{r-2}\right| \int_{0}^{1}\left|1+t_{r-2}+t_{r-3}\right| \cdots \\
& \left.\cdots \int_{0}^{1}\left|1+t_{2}+t_{1}\right| d t_{1} \cdots d t_{r-3} d t_{r-2} d t_{r-1}\right) \\
\leq & \epsilon\left(1+\lambda H+(\lambda H)^{2}+\ldots+(\lambda H)^{r-1}\right)=\epsilon\left(\frac{1-(\lambda H)^{r}}{1-\lambda H}\right)
\end{aligned}
$$


as $r \rightarrow \infty$, we obtain

$$
\left|\phi^{*}(x)-\psi^{*}(x)\right| \leq \frac{1}{1-\lambda H} \epsilon .
$$

Let us choose $S=\frac{1}{1-\lambda H}$, hence $\left|\phi^{*}(x)-\psi^{*}(x)\right| \leq S \epsilon$, and $0<\lambda H<1$, where $S$ is the Hyers-Ulam stability constant for (2). Hence, by the virtue of Definition 4 the non-homogeneous Fredholm integral equation (2) has the Hyers-Ulam stability.

Finally, the following corollary proves the Hyers-Ulam-Rassias stability of the non-homogeneous Fredholm integral equation of second kind (2).

Corollary 10. Consider $H$ a fixed real number such that $H \geq \frac{5}{2}$ and $\lambda H<1$. Let $\phi:[0,1] \rightarrow \mathbb{R}$ a continuous function and the kernel $K:[0,1] \times[0,1] \rightarrow \mathbb{R}$ defined by $K(x, t)=1+x+t$ such that

$$
\int_{0}^{1}|1+x+t| \theta(t) d t \leq \theta(x) \int_{0}^{1}|1+x+t| d t,
$$

for all $x \in[0,1]$, where $\theta \in C\left(\mathbb{R}_{+}, \mathbb{R}_{+}\right)$. If $\phi$ is such that

$$
\left|\phi(x)-x-\lambda \int_{0}^{1}(1+x+t) \phi(t) d t\right| \leq \theta(x),
$$

then there exists a solution $\psi:[0,1] \rightarrow \mathbb{R}$ of the non-homogeneous Fredholm integral equation (2) and a real constant $S$ such that $|\phi(x)-\psi(x)| \leq S \theta(x)$ for all $x \in[0,1]$.

\section{Examples}

In order to illustrate our results we will present some examples.

Let us consider the non-homogeneous Fredholm integral equation of second kind (2) defined by

$$
\phi(x)=x+\lambda \int_{0}^{1}(1+x+t) \phi(t) d t
$$

for all $x \in[0,1]$ and $\lambda=\frac{1}{5}$. Let $H=\frac{13}{5}$ and the perturbation of the solution $\varphi(x)=$ $\frac{587}{500} x+\frac{28}{100}$.

We realize that all conditions of Theorem 9 are satisfied. In fact $\lambda H=\frac{13}{25}<1$ and $\varphi$ is a continuous function such that

$$
\left|\phi(x)-x-\lambda \int_{0}^{1}(1+x+t) \phi(t) d t\right|=\left|\frac{3}{5000} x+\frac{1}{3000}\right| \leq \frac{7}{7500}:=\epsilon .
$$

By the exact solution $\psi(x)=\frac{210}{179} x+\frac{50}{179}$, we realize that

$$
|\phi(x)-\psi(x)|=\left|\frac{73}{89500} x+\frac{3}{4475}\right| \leq \frac{1}{1-\lambda H} \epsilon=\frac{7}{3600} .
$$

To illustrate the inequality (10), we have the Figure 1

Let us consider the same non-homogeneous Fredholm integral equation of second kind (2) but now with $\lambda=\frac{1}{100}$. Let $H=3$ and the perturbation of the solution $\varphi(x)=$ $\frac{10052}{10000} x+\frac{851}{100000}$. We have $\lambda H=\frac{3}{100}<1$ and $\varphi$ a continuous function such that

$$
\left|\phi(x)-x-\lambda \int_{0}^{1}(1+x+t) \phi(t) d t\right|=\left|\frac{5334}{60000000} x+\frac{341}{60000000}\right| \leq \frac{227}{2400000}:=\epsilon .
$$




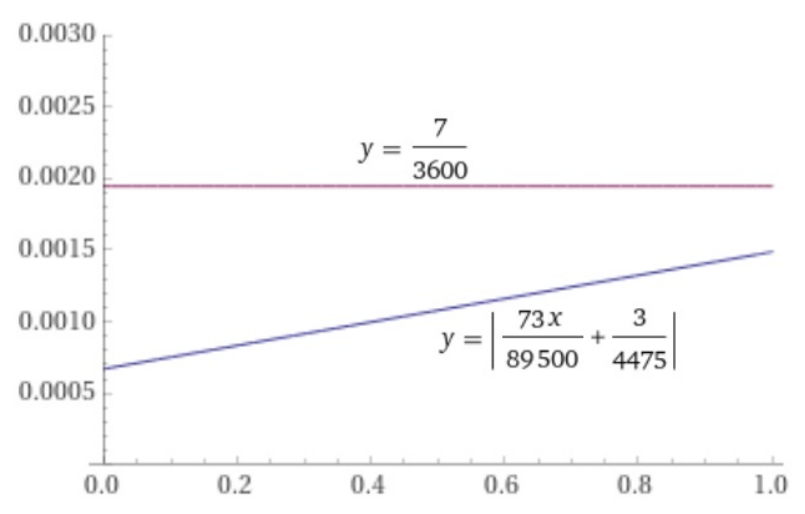

Figure 1:

By the exact solution $\psi(x)=\frac{118200}{117599} x+\frac{1000}{117599}$, we realize that

$$
|\phi(x)-\psi(x)|=\left|\frac{26287}{293997500} x+\frac{76749}{11759900000}\right| \leq \frac{1}{1-\lambda H} \epsilon=\frac{227}{2328000} .
$$

If we consider $H=30$, we get a worse result but still acceptable. We get,

$$
|\phi(x)-\psi(x)|=\left|\frac{26287}{293997500} x+\frac{76749}{11759900000}\right| \leq \frac{1}{1-\lambda H} \epsilon=\frac{227}{1680000} .
$$

So we have that the non-homogeneous Fredholm integral equation of second kind (2) has the Hyers-Ulam stability.

To illustrate the inequalities $(11)$ and $(12)$, we have the Figure 2

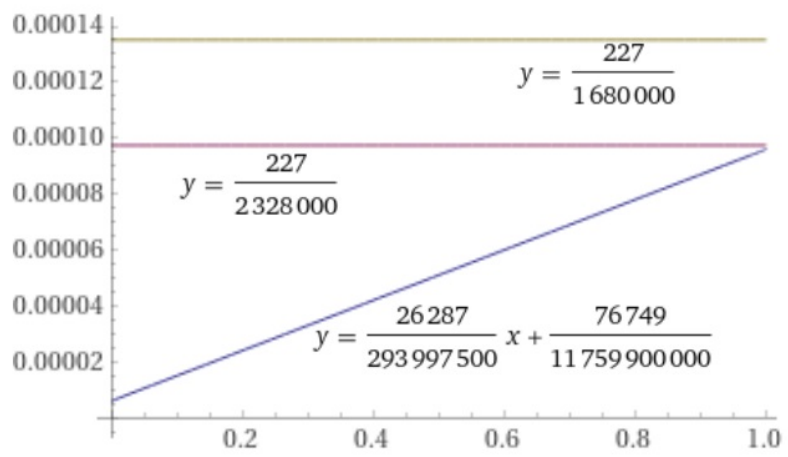

Figure 2:

\section{Acknowledgment}

Research supported by the Center of Mathematics and Applications of University of Beira Interior (CMA-UBI) through the Portuguese Foundation for Science and Technology (FCT - Fundação para a Ciência e a Tecnologia), under the reference UIDB/00212/2020, and by the Center for Research and Development in Mathematics and Applications (CIDMA) through the Portuguese Foundation for Science and Technology (FCT - Fundação para a Ciência e a Tecnologia), under the reference UIDB/04106/2020. 


\section{References}

[1] Alsina C, Ger R. On Some inequalities and stability results related to the exponential function. Journal of Inequalities Applications 1998; 2 (4): 373-380.

[2] Andras S, Baricz A, Pogány T. Ulam-Hyers stability of singular integral equations, via weakly Picard operators. Fixed Point Theory 2016; 17 (1): 21-36.

[3] Aoki T. On the stability of the linear transformation in Banach Spaces. Journal of the Mathematical Society of Japan 1950; 2 (1-2): 64-66.

[4] Başci Y, Öğrekçi S, Misir A. On Ulam's type stability criteria for fractional integral equations including Hadamard type singular kernel. Turkish Journal of Mathematics 2020; 44 (4): 1498-1509.

[5] Belbali H, Benbachir M. Existence results and Ulam-Hyers stability to impulsive coupled system fractional differential equations. Turkish Journal of Mathematics 2021; 45: 1368-1385.

[6] Bourgin DG. Classes of transformations and bordering transformations. Bulletin of the American Mathematical Society 1951; 57 (4): 223-237.

[7] Castro LP, Simões AM. Different Types of Hyers-Ulam-Rassias Stabilities for a Class of Integro-Differential Equations. Filomat 2017; 31 (17): 5379-5390.

[8] Castro LP, Simões AM. Hyers-Ulam and Hyers-Ulam-Rassias stability of a class of Hammerstein integral equations. American Institute of Physics, Conference Proceedings 2017; 1798 (1): 1-10.

[9] Castro LP, Simões AM. Hyers-Ulam-Rassias stability of nonlinear integral equations through the Bielecki metric. Mathematical Methods in the Applied Sciences 2018; 41 (17): 7367-7383.

[10] Castro LP, Simões AM. Hyers-Ulam and Hyers-Ulam-Rassias Stability for a Class of Integro-Differential Equations. Mathematical Methods in Engineering: Theoretical Aspects. Tas K, Baleanu D, Tenreiro Machado JA (edts.), Springer, 2019; 81-94.

[11] Chung J. Hyers-Ulam stability theorems for Pexiders equations in the space of Schwartz distributions. Archiv der Mathematik 2005; 84 (6): 527-537.

[12] Gachpazan M, Baghani O. Hyers-Ulam stability of Volterra Integral equation. International Journal of Nonlinear Analysis and Applications 2010; 1(2); 19-25.

[13] Gachpazan M, Baghani O. Hyers-Ulam stability of Nonlinear Integral Equation. Fixed Point Theory and Applications 2010; 2010: 1-6.

[14] Gu Z, Huang J. Hyers-Ulam stability of Fredholm Integral equation. Mathematica Aeterna 2015; 5(2): 257-261.

[15] Hua L, Huang J, Li Y. Hyers-Ulam stability of some Fredholm Integral equation. International Journal of Pure and Applied Mathematics 2015; 104 (1): 107-117.

[16] Hyers DH. On the stability of the linear functional equation. Proceedings of the National Academy of Sciences of the United States of America 1941; 27 (4): 222-224. 
[17] Li T, Zada A, Faisal S. Hyers-Ulam stability of nth order linear differential equations. Journal of Nonlinear Science and Applications 2016; 9 (5): 2070-2075.

[18] Luo D, Abdeljawad T, Luo Z. Ulam-Hyers stability results for a novel nonlinear Nabla Caputo fractional variable-order difference system. Turkish Journal of Mathematics 2021; 45 (1): 456-470.

[19] Murali R, Selvan AP. On the Generlized Hyers-Ulam Stability of Linear Ordinary Differential Equations of Higher Order. International Journal of Pure and Applied Mathematics 2017; 117 (12): 317-326.

[20] Murali R, Selvan AP. Hyers-Ulam-Rassias Stability for the Linear Ordinary Differential Equation of Third order. Kragujevac Journal of Mathematics 2018; 42 (4): 579-590.

[21] Murali R, Selvan AP. Hyers-Ulam stability of nth order differential equation. Proyecciones 2019; 38 (3): 553-566.

[22] Murali R, Selvan AP. Fourier Transforms and Ulam Stabilities of Linear Differential Equations. Frontiers in Functional Equations and Analytic Inequalities, Springer, Cham 2019; 195-217.

[23] Murali R, Selvan AP, Park C. Ulam stability of linear differential equations using Fourier transform. AIMS Mathematics 2020; 5 (2): 766-780.

[24] Rassias JM, Murali R, Selvan AP. Mittag-Leffler-Hyers-Ulam Stability of Linear Differential Equations using Fourier Transforms. Journal of Computational Analysis and Applications 2021; 29 (1): 68-85.

[25] Rassias TM. On the stability of the linear mappings in Banach Spaces. Proceedings of the American Mathematical Society 1978; 72 (2): 297-300.

[26] Rassias TM. On the stability of functional equations and a problem of Ulam. Acta Applicandae Mathematicae 2000; 62 (1): 23-130.

[27] Ravi K, Murali R, Selvan AP. Stability for a Particular Fredholm Integral equation. Asian Journal of Mathematics and Computer Research 2016; 11 (4): 325-333.

[28] Ravi K, Murali R, Selvan AP. Hyers-Ulam Stability of a Fredholm Integral equation with Trigonometric Kernels. Universal Journal of Mathematics 2016; 1 (1): 24-29.

[29] Rus IA. Ulam Stabilities of Ordinary Differential Equations in a Banach Space. Carpathian Journal of Mathematics 2010; 26 (1): 103-107.

[30] Takahasi SE, Miura T, Miyajima S. On the Hyers-Ulam stability of the Banach space-valued differential equation $y^{\prime}=\lambda y$. Bulletin of the Korean Mathematical Society 2002; 39 (2): 309-315.

[31] Ulam SM. A Collection of Mathematical Problems. Interscience Publishers, New York, 1960.

[32] Xue J. Hyers-Ulam stability of linear differential equations of second order with constant coefficient. Italian Journal of Pure and Applied Mathematics 2014; 32: 419-424. 